\title{
Results on magnetic field inside and outside the magnetosphere $(*)$
}

\author{
F. Mariani $(* *)$ \\ Ricevuto il 20 Aprile 1966
}

Summary. - Present knowledge on the magnetic field of celestial bodies in briefly reviewed.

Theoretic results on the interaction of the solar wind and its frozenin magnetic field with the geomagnetic field are presented. Satellite measurements within and outside the magnetosphere are then discussed: agreement with theoretical results is quite good.

Rrassunto. - Si illustrano brevemente le attuali conoscenze sui campi magnetici dei vari corpi celesti nello spazio interplanetario.

Dopo un cenno agli effetti teoricamente prevedibili sulla interazione del vento solare e del campo magnetico in esso "congelato" con il campo magnetico terrestre, si presentano i più recenti risultati delle misure a bordo di satelliti entro e fuori la magnetosfera. L'accordo tra risultati sperimentali e tecnici è, nelle linee generali, abbastanza buono.

\section{INTRODUCTION}

Our knowledge of magnetic fields in interplanetary space has rapidly increased in recent years. From direct measurements of magnetic fields and related theoretical studies we now have some general ideas on the structure of the magnetic field between the Earth and the Sun.

It is the purpose of this talk to present a survey of the recent, most important, space experiments and related theoretical results concerning the magnetic field of interplanetary space.

First of all we will briefly consider the physical state of the interplanetary medium from a theoretical point of view; after that we shall review the experimental results which are presently available.

(*) This is an abstract of a lecture given at ESTEC (European Space Technology Centre) in Noordwijk on December 17th, 1965.

(**) Istituto di Fisica - Universitì di Roma. Gruppo SPAROM-GIFCO del C.N.R. - Roma - Italy. 


\section{THE MIGNETIC FIELIDS OF CELESTIAL BODIES}

\subsection{The Earth's magnetic field.}

The Earth's magnetic field is due primarily to internal sources and is approximately expressed as a dipole field. It can be represented as the gradient of a potential function $V$, given by a spherical harmonics series, the coefficients of which can be computed if sufficient knowledge of the surface magnetic field is available. This function is expressed by:

$$
Y=R_{e} \sum_{n=1}^{e}\left(\frac{T_{e}}{r}\right)^{n+1} \sum_{m=0}^{n} P_{n}^{m}(\cos \vartheta)\left[g_{\imath}^{m} \cos m \psi+h_{,}^{m} \operatorname{sen} m \psi\right]
$$

where $R_{c}$ is the earth's radius $(6370 \mathrm{~km}), v$ the geocentric distance of the point at which $V$ is determined, $\vartheta$ the geographic colatitude, $\psi$ the longitude east of this point, $P_{n}(\cos \vartheta)$ the nomalized Legendre polynomial of degree $n$ and order $m, g_{n}^{m}$ and $h_{n}^{m}$ the numerical coefficients to be determined $\left(^{1}\right)$.

Tables of the above coefficients lave been computed by Fricu and

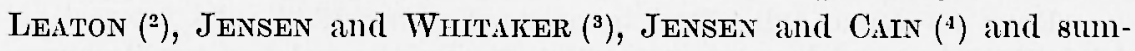
marized by Heuring $\left(^{5}\right)$. The first three coefficients $g_{1}^{0}, g_{1}^{1}$ and $h_{1}^{1}$ of the equation [1] lead to the familiar axial dipole field potential:

$$
V=\frac{M \cos \vartheta}{r^{2}}=\frac{\vec{M} \cdot \vec{r}}{r^{3}}
$$

where:

$$
\begin{aligned}
& g_{1}^{0}=0,304112 \text { gauss } \\
& g_{1}^{1}=0,021474 " \text { according to Jensen and Cain } \\
& h_{1}^{\mathbf{1}}=-0,057989 \text { » } \\
& M \cong 8.10^{25} \text { gauss } \times \mathrm{cm}^{3} \\
& \text { geomagnetic north pole at } 79^{\circ} \text { North Lat., } 290,3^{\circ} \text { Est Long. } \\
& \text { " south pole at } 79^{\circ} \text { South Lat., } 110,3^{\circ} \text { Est Long. }
\end{aligned}
$$

The left-hand part of Fig. 1 shows the lines of the field in a vacum interplanetary space.

A second contribution to the mannetic potential, of the order of a few percent of [1], is due to external sources (e.g. electric currents in the ionosphere or in the exosphere, probably up to distances of some Earth's radii). 
Diurnal, seasonal and solar cycle variations are superimposed on the "static" field; moreover, typical, irregular variations - the socalled $D_{s t}$ variations - occur during perturbed conditions (magnetic storms). The order of magnitude of these effects amounts to tens up to many hundreds of gammas (1 gamma is $10^{-5}$ gauss).

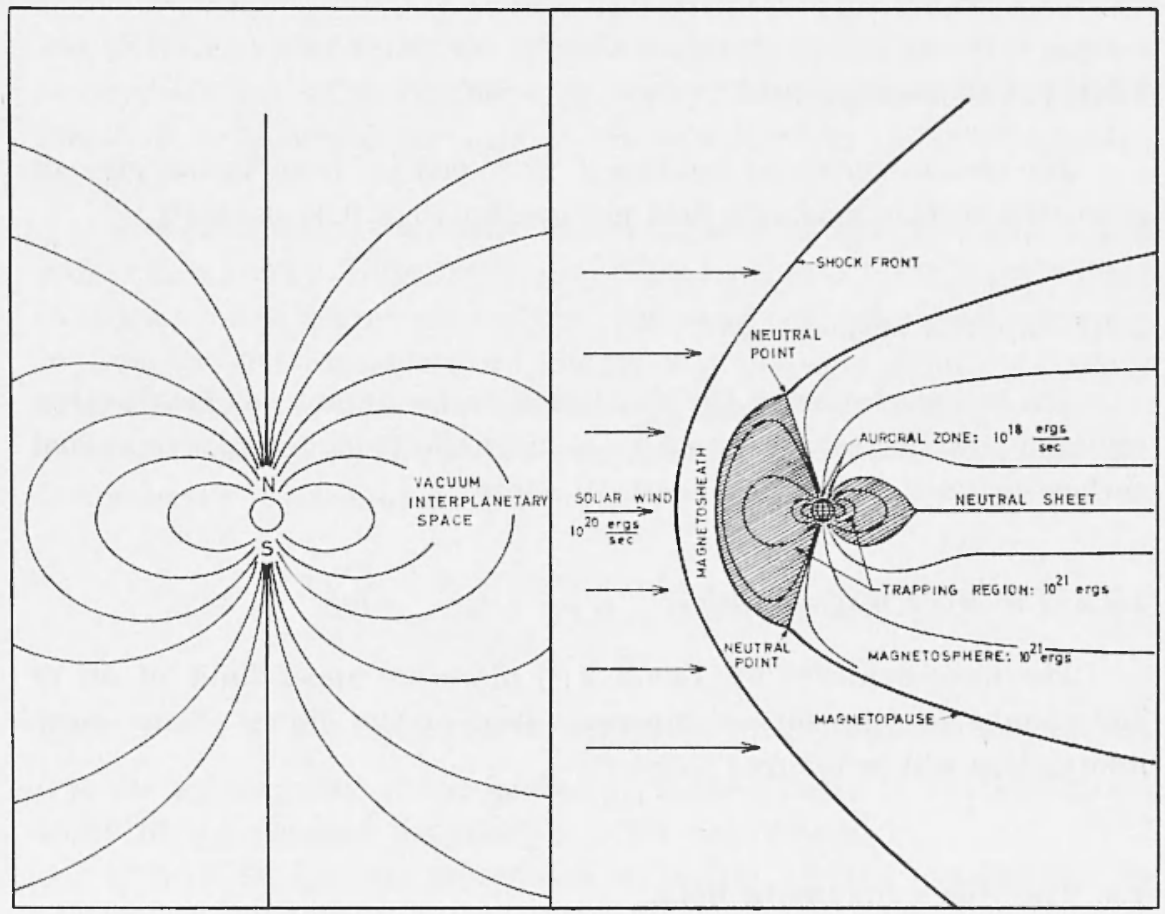

Fig. 1 - Comparison of the sketches of the magnetosphere in 1950's and in the early 1960 's (this is a mixture of experimental facts and theoretical predictions). Typical energy fluxes and energy content are indicated.

The behavior of the magnetic field during a magnetic storm is particularly interesting: a sudden increase of the horizonthal component $H$, all over the earth, occurs within a few seconds everywhere; a first phase, a few hours long, during which $\not H$ is above its normal, value followed by a main phase characterized by lower $H$ values. The initial phase of the magnetic storm was attributed by Chapman and Ferraro to an incoming solar plasma stream. After that a diamagnetic ring current gradually builds up on the equatorial plane at distances of a few earth's radii; the recovery phase of the magnetic storm is the result of the dissipation of the magnetic energy associated to this ring current. 


\subsection{The magnetic field of the other planets and the Moon.}

No direct information is presently available on the existence of possible magnetic fields of the other planets (or satellites), with the exception of Venus, Jupiter and the Moon.

\subsubsection{Venus magnetic field.}

The measurements by Mariner 2, at $35.000 \mathrm{~km}$ from Venus, give an estimated surface magnetic field not greater than 0,05 gauss ${ }^{8}$ ).

\subsubsection{Jupiter's magnetic field.}

The interpretation of the radioemission by Jupiter, as synchrotron radiation by electrons trapped in a magnetic ficld gives an estimated surface magnetic field of at least 10 gauss $\left({ }^{7}\right)$.

\subsubsection{The Moon magnetic field.}

The measurements by Lunik $2\left(^{8}\right)$ place an upper limit of 50 to 100 gamma for the surface magnetic field of the Moon. Some more information will be referred to later.

\subsection{The Sun's magnetid field.}

As regards the Sun, on the basis of observations of the longitudinal Zeeman effect, a photospheric field has been found with an average intensity of the order of 1 gauss and peak values of a few gausses $\left(^{9}\right)$; this result is in contrast with the earlier estimate of a general field of 50 gauss given by HALE $\left({ }^{10}\right)$. The direction of the photospheric field is variable and a reversal of both south polar and north polar fields has been observed in Spring 1957 and in Autumn 1958, respectively.

In addition, very strong fields, up to thousands of gausses, in a rather small scale $\left(10^{4}\right.$ to $\left.10^{5} \mathrm{~km}\right)$, occur near the sunspots, the active regions of the Sun. Both bipolar and unipolar fields are observed; in the first case, the magnetic field lines return to the Sun within the general boundaries of the same region; in the second case, these lines return, in a diffusive manner, over a large portion of the solar disk. 


\subsection{Magnetic field of interplanetary space.}

The magnetic fields considered in the preceding paragraphs cannot give an appreciable magnetic intensity throughout the solar system. In fact, the general magnetic field of the Sun becomes only $10^{-2}$ gamma at one astronomical unit; a similar decrease occurs for the sunspot fields due to their smaller scale; the Earth's magnetic field is also $10^{-2}$ gamma at only $10^{6} \mathrm{~km}$. On this basis no appreciable magnetic fields should therefore be expected throughout the solar system (in interplanetary space).

However, recent magnetic observations with satellites and space probes give a very different picture. This "paradox" can be explained as follows. As a consequence of the ejection of charged particles (mainly protons and electrons, a stream known as "the solar wind" (plasma)) from the Sun, the solar magnetic field is carried out from the Sun.

Infact, from the Maxwell's equations of electrodynamics one can easily derive the following equation:

$$
\left.\frac{\partial \vec{B}}{\partial t}=\frac{1}{4 \pi \mu \sigma} \nabla^{2} \vec{B}+\vec{\nabla} \times \overrightarrow{(v} \times \vec{B}\right)
$$

where $\vec{B}=\mu \vec{H}(\vec{B}$ is the magnetic induction; $\vec{H}$ the magnetic field vector; $\mu$ is the permeability of the medium; $t$ is the time; $\vec{v}$ is the vector velocity of the charged particles; $\sigma$ is the conductivity).

If $\sigma \rightarrow \infty$, i.e. the material is a perfect electric conductor, the term containing $\overrightarrow{\nabla=B}$ is negligible and straightforward use of equation [3] leads, by use of the Stokes theorem, to a null time-derivative of the magnetic flux $\Phi(\vec{B})$ through any closed line in the magnetic field. In other words the magnetic flux through a closed contour is conserved, no matter how it deforms with time. This means that, due to the high electric conductance the magnetic field lines are "frozen-in" and they move with the solar wind out of the Sun. On the other hand, one edge of each line of force is rigidly connected with the solar region where it originated and co-rotates with the Sun with the typical period of about 27 days as seen from the Earth. As a consequence, if the magnetic lines are originally directed along the radius, with respect to the Sun, they assume a different configuration as viewed from the Earth. In particular, they depend strongly on the plasma velocity $\vec{v}$. 
1.4.1 The Parker model.

An exact computation by PARKer $\left({ }^{11}\right)$ in the steady case gives, for the three components of $\vec{B}$, at a point in space:

$$
\left.\begin{array}{l}
\left.B_{r}=B_{r} R, \vartheta, \Phi-r \frac{\Omega}{v}\right) \frac{R^{2}}{r^{2}} \\
B_{\vartheta}=0 \\
B_{\Phi}=B_{r}\left(R, \vartheta: \Phi-r \frac{\Omega}{v}\right) \frac{\Omega}{v} \frac{R^{2}}{r}
\end{array}\right\}
$$

where $R$ is the radius of the Sun and $\Omega$ its angular velocity; $r, \vartheta, \Phi$ are polar coordinates in a heliocentric system (with $\vartheta=0$ at the Sun equator).

The magnetic field lines are shown in Fig. 2, for the case $v=$ $300 \mathrm{~km} / \mathrm{sec}$. The angle $\psi$, defined by:

$$
\psi=\tan ^{-1}\left(\frac{\Omega r}{v}\right)
$$

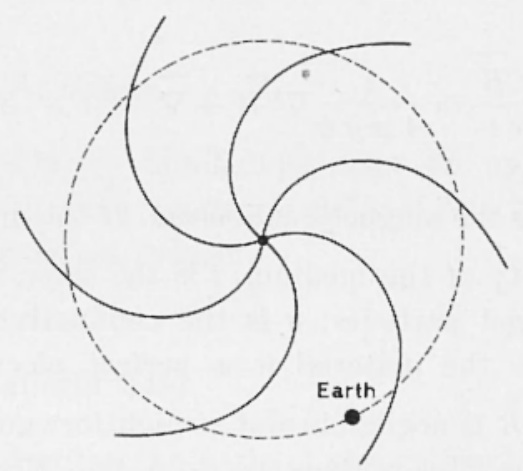

Fig. 2 - The lines of force of the quiet-day interplanetary magnetic field near the Earth resulting from extension of the general solar field by an idealized uniform $300 \mathrm{~km} / \mathrm{sec}$ quiet-day solar wind (Parker model).

is the "streaming angle", i.e. the angle between the plasma velocity vector and the magnetic field line at a point of coordinate $r$; this angle is the well known "garden hose" angle of Chapman anf Bartels.

At the Earth the streaming angle for the case $v=300 \mathrm{~km} / \mathrm{sec}$ is $\psi \approx 56^{\circ}$. The angle $\psi$ tends to the limit $\psi=\frac{\pi}{2}$ when $r$ increases to infinity, which means that the magnetic field lines approach more and more the transverse direction. 
As regards the polarity of the magnetic field, in the above model of streaming lines of force this field can be either away or toward the Sun. If two tubes of magnetic flux contain lines of opposite polarity a neutral point or surface can be originated ( $\left.{ }^{12}\right)$.

\subsubsection{The Gold Model.}

A different, but complementary view is expressed by a time-dependent model first proposed by Cocconi, Greisen, Morrison, Gold and Hayarawa $\left({ }^{13}\right)$ and more recently by Piddington $\left({ }^{14}\right)$. According to this model a continuous transient procession of tongues of magnetic lines of force takes place from the perturbed chromosphere through the corona and the interplanetary medium (Fig. 3). Both the edges of

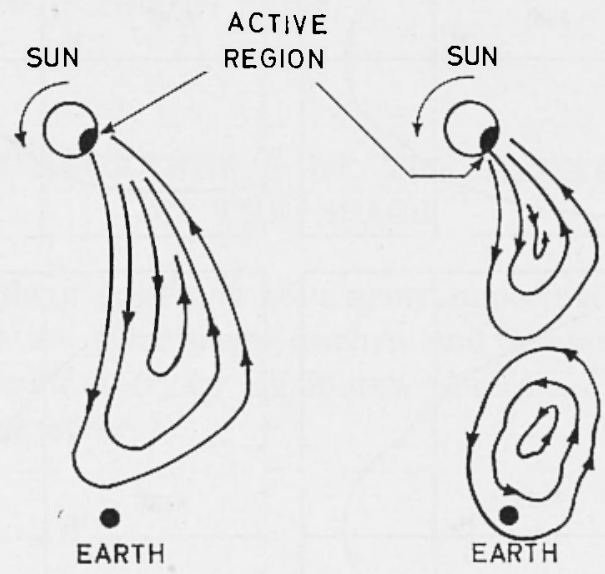

Fig. 3 - The lines of force of the interplanetary magnetic field in the Gold model.

each line of force are anchored into the chromosphere, even though they become more and more elongated. Occasionally, some instability can sever a tongue which thus far becomes a magnetic bulge containing a neutral point and closed lines of force. This magnetic cloud then propagates in the interplanetary medium as an independent entity.

\subsubsection{The magnetosphere and cavities.}

A last general question is the ultimate fate of the solar plasma and the associated magnetic field: if, near the Sun, the dynamic pressure 
of the solar plasma $\frac{1}{2} \varrho v^{2}$, is much greater than the magnetic pressure $B-/ 8 \pi$, the solar plasma can actually leave the Sun together with its frozen-in magnetic field. The motion lasts until the two pressures become equal: then, large disturbances affect the solar wind and the magnetic field. $\Lambda$ situation of this kind appears to occur as soon as the solar wind reaches within a few radii of the Earth. Due to its high velocity, the plasma is hypersonic and a skock wave develops.
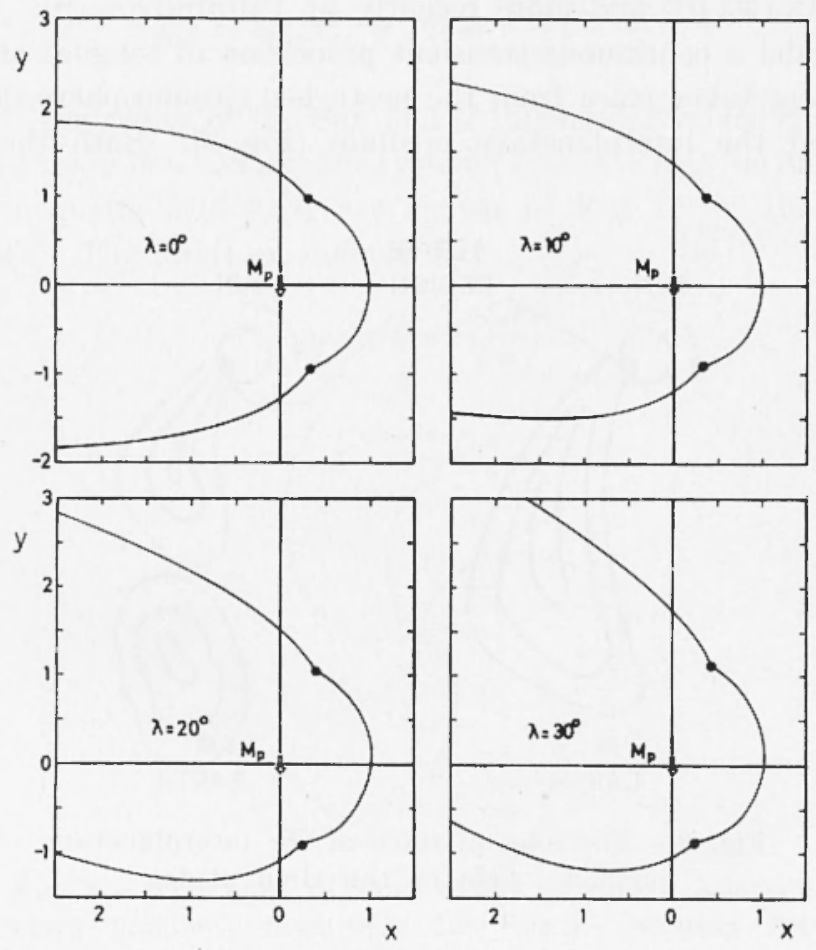

DISTANCES IN EARTH RADII

- neutral points

Fig. 4 - Form of the boundary of the hollow in the meridian plane containing the dipole axis and the Sun-Earth line; $0^{\circ} \leqslant \lambda \leqslant 30^{\circ}: \lambda$ is the angle between the direction of the incident plasma and the normal to the axis of the dipole. Distances $x$ and $y$ are in units of $r_{o}$, where $r_{0}$ is the geocentric distance of magnetopause on the equatorial plane when $\lambda=0^{\circ}$ (after Spreiter and Briggs).

At some distance ahead of this skock wave, the geomagnetic field boundary is sufficiently strong to reflect the incoming plasma. As a 
result, the flow of the plasma stream is around the Earth and a sort of a hole is carved within the plasma stream (the so-called geomagnetic cavity or magnetosphere (Fig. 4). In a transition region, or magnetosheath, between the shock wave and the boundary of the magnetosphere of the order of about 4 Earth radii, the geomagnetic field is compressed on the side of the Sun and depressed on the other side (right hand of Fig. 1). In the normal conditions the boundary which separates the quiet magnetosphere from the magnetosheath is called magnetopause. In the transition region, which is turbulent, the magnetic field is irregular. The magnetosphere contains a highly conductive ionized gas so that indirect effects can be expected there, as a consequence of events occurring at the boundary or in the transition region.

Similar magnetic cavities can obviously be expected around other magnetized planets or celestial bodies.

\section{DIRECT MEASUREMENTS OF THE MAGNETIC FIELD IN THE SPACE}

At present, there have not been many opportunities to measure magnetic fields in the outer magnetosphere and in interplanetary space although some results are very significant. These are summarized in the following paragraphs.

\subsection{Pioneer 1 .}

The first measurements were made by Pioneer $1\left({ }^{15}\right)$. However, the use of rotating coil magnetometer, which limits measure only to the component $B \perp$ normal to the spin axis of the vehicle, greatly affects the physical meaning of the results. Anyway, large fluctuations over small distances were detected at geocentric distances greater than twelve Earth radii.

\subsection{Lunik 2 and Pioneer V.}

The measurements with Lunik $2\left(^{8}\right)$ on the existence of the interplanetary magnetic field were only qualitative, due to the limited $\mathrm{dy}$ namic range of the magnetometer used (triaxial saturable core magnetometer). 
Better results were obtained with Pioneer 5 (16, 17). Although the normal component $B_{\perp}$ was measured, three different regions could be clearly defined: an inner quiet region, an intermediate fluctuating region and an outer region where a quiet field is present with an intensity well above the expected residual geomagnetic field. A close correlation between Pioneer 5 data and the Earth measured $a_{p}$ index is apparent ${ }^{18}$ ): particularly, in correspondence to the s.c. magnetic storm observed on March 30, 1960. An ablupt change of $B$ from a few gammas to more than 20 gammas took place and was detected by the probe. Using the same data, also a direct relation between the flare activity on the Sun and the subsequent occurrence of magnetic transients at the location of Pioneer 5 was found $\left({ }^{19}\right)$, implying an average propagation velocity of about $1000 \mathrm{~km} / \mathrm{sec}$.

\subsection{Most significant results.}

Are those obtained with Explorer $10\left({ }^{20}\right)$, Explorer $12\left({ }^{21}\right)$, Explorer $14\left({ }^{22}\right)$ and Explorer $18\left({ }^{23}\right)$ : we will consider them in some detail.

\subsubsection{Explorer 10 .}

This satellite gave the first reliable vector magnetic measurements by means of $R b$ vapor and two fluxgate magnetometers; unfortunately, a surface contamination of the passive thermal control of the $R b$ magnetometer limited measurements strongly and all results at distances above about $8 R_{e}$ had to be obtained by means of the fluxgate magnetometer. This satellite also carried a plasma probe to measure selected energy ranges of the proton fluxes.

The results can be best interpreted by taking into account the coordinate reference system shown in Fig. 5 .

As regards the geomagnetic reference field, the computed Finch and Leaton coefficients have been assumed. Typical variations of the total field intensity $F$ and the angles $\vartheta(\vartheta=0$ on the ecliptic plane $)$ and $\Phi(\Phi=0$ in the direction towards the Sun, $\Phi=180$ in the antisolar direction) are shown in Fig. 6. Total intensity up to about 30 gammas occur, as well as large and irregular variations of the angles $i$ and $\Phi$. However, some steady intervals lasting about one hour can be found. Some of the main conclusions that can be drawn are as follows:

a) at geocentric distances $r$ between $r_{1}=11,5 R_{e}$ and $22 R_{e}$, the total magnetic field intensity $F(r)$ is approximated by the expression:

$$
F(r)=F\left(r_{1}\right)\left(r_{1} / r\right)^{x}
$$




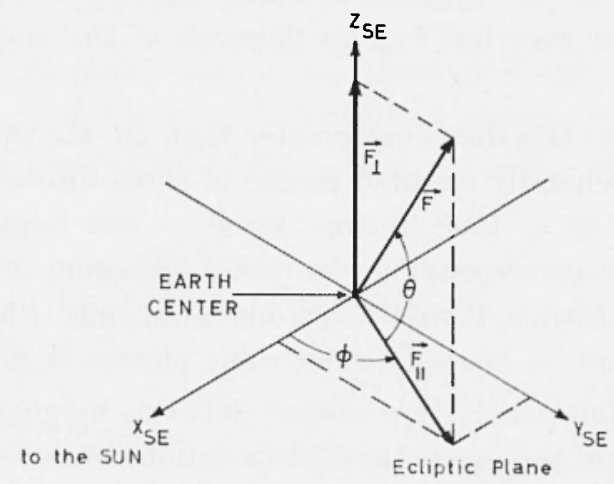

Fig. 5 - Definition of solar-ecliptic coordinates system: $X_{S E}$ is from the satellite to the Sun, $Y_{S E}$ in the ecliptic plane, $Z_{S E}$ is normal to this plane so that $X_{S F}, Y_{S E}, Z_{S E}$ is a right-handed system.

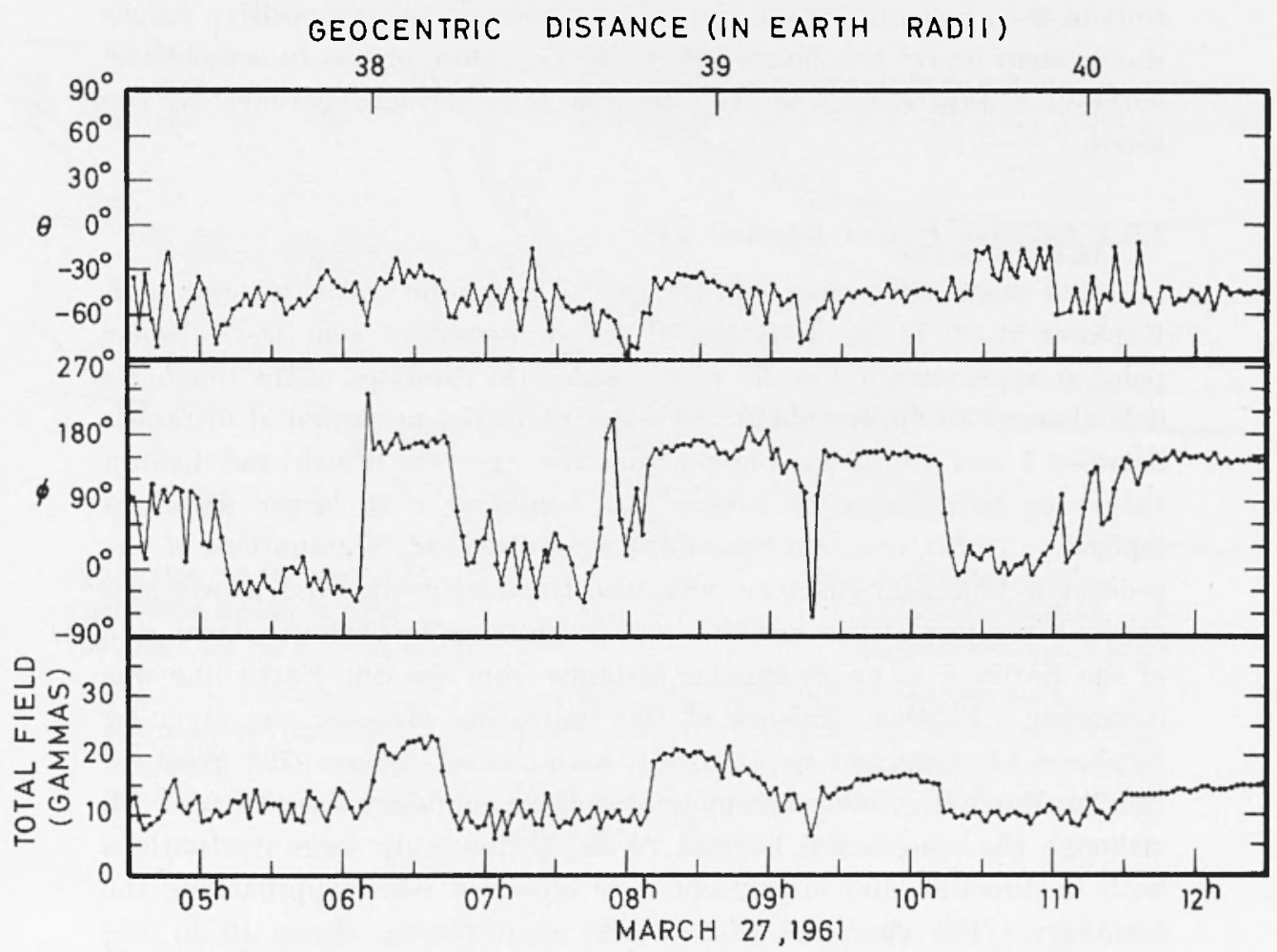

Fig. ( ) - Magnetic field measurements by Explorer 10 on 27 March 1961 (after Heppner et al.). 
where the value of the exponent $x$ is found between 0,6 and 1,0 . This expresses a rather slow but regular decrease of the magnetic field with distance;

b) at geocentric distances greater than $22 R_{e}$, the magnetic lines of the field are generally oriented in one of three directions: each being, centered around $\Phi=180^{\circ}$ (region $\Lambda$ ), $\Phi=90^{\circ}$ (region $\left.\mathrm{B}\right), \Phi=0^{\circ}$ (region $\mathrm{C}$ ), with corresponding distinctly different levels of average field intensities. Region $\mathrm{B}$ and $\mathrm{O}$ are observed only when solar plasma is detected; region $A$, instead, when solar plasma is not detected;

c) a possible correlation occurs between magnetic field changes at the satellite and the magnetic field variations observed on the Earth at a number of observatories;

d) an appreciable and rather quick increase of the field intensity (from 10 to 20 gammas) occurred 0 to 7 minutes after a sudden magnetic commencement (s. c.) at the Earth; as regards the direction, an abrupt shift of $\Phi$ to zero and a tendency of $\vartheta$ to increase toward positive values was evident about two hours before the s.c., more or less in coincidence with the abrupt change of the magnetic field at high latitudes on the Earth.

\subsubsection{Explorer 12 and Explorer 14.}

The most interesting feature of the magnetic measurements with Explorer $12\left({ }^{21}\right)$ is the detection of a thin boundary near the subsolar point at approximately $10 R_{e}$ within which the direction of the magnetie field changes abrouptly about $180^{\circ}$; the intensities measured at distances between $\tau$ and $10 R_{c}$ were larger that the expected Finch and Leaton values up to a factor of 2 near the boundary. At larger distances (apogee $=13 R_{e}$ ), irregular variations were observed. Fluctuations of the geocentric boundary distance were also frequently observed, as well as a gradual increase as the satellite was progressing toward the dawn side of the Earth, i. e. as its angular distance from the Sun-Earth line was increasing. Further evidence of this increasing distance was given by Explorer 14, launched in an orbit with initial apogee $70^{\circ}$ West of the Sun-Earth line. Often no magnotospheric boundary was observed $\left({ }^{22}\right)$, although the apogee was beyond $16 R_{c}$. Increasingly large fluctuations both in direction and magnitude were observed when approaching the boundary. The character of the field at distances above $10 R_{e}$ was generally that of a steady compressed greomagnetic field at a level of about 50 gammas. 


\subsubsection{Explorer 18 (IMP-1).}

The most interesting results are those of Explorer $18\left({ }^{23}\right)$, which gave continuous and successful information during about fifty orbits ( $\sim 6$ months). The initial dayside, very eccentric, orbit was subiect



Fig. 7 - Interpretation of the IMP-1 magnetic field measurements of the cislunar environment. Illustrated are the directions of the interplanetary magnetic field, the position of the magnetosphere boundary and the collisionless shock wave as observed by the magnetic field experiment (after Ness et al.).

to a progressive rotation with respect to the Sun: the angle between the line of apsides and the sun-earth radius vector was $25^{\circ}$ at the launching and became $176^{\circ}$ on May 3, 1964 (Fig. 7). Both day- and night- 
sides of the Earth were explored at distances up to 32 Earth radii. Typical variations of the magnetic elements $F, \dot{\psi}, \Phi$ are shown in Fig. 8 for orbit N. 15 on January 21, 1964. The lower diagrams give the

(15)
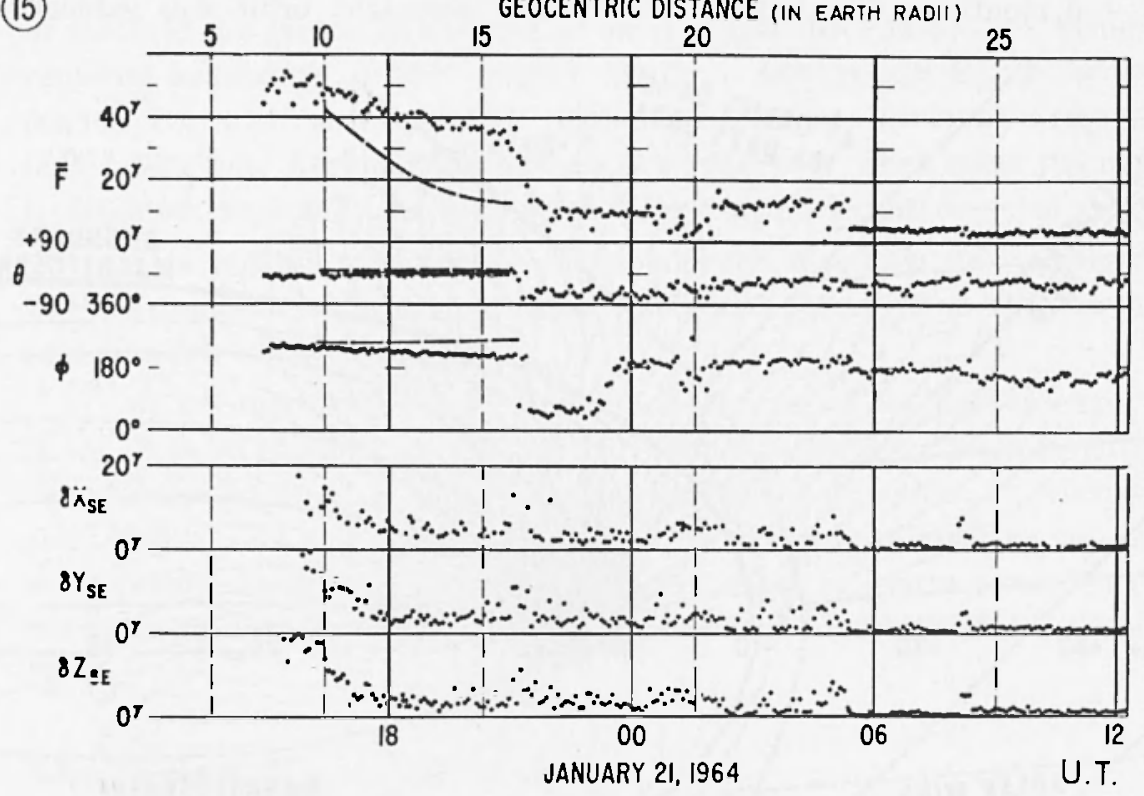

Fig. 8 - Magnetic field data from orbit No 15 (January 21, 1964) illustrating outbound traversal of magnetopause boundary at $15,7 R_{e}$, wave boundary at $22,7 R_{e}$ and precursor at $24,5 R_{e}$ (after Ness et il.).

"variances" $\delta X_{S E}, \delta X_{S E}$ and $\delta Z_{S E}$, i.e. the standard deviations of the components $X_{S E}, Y_{S E}$ and $Z_{S E}$ of the magnetic field with respect to their averages on each sampling time interval of 5,46 minutes.

In practice, similar characteristics are displayed in each orbit. Again, three regions are clearly identified:

a) a region (magnetosphere) where the geomagnetic field is contained and more increased with respect to the expected intensities inferred by use of [1];

b) a transition region, abruptly starting at $15,7 R_{e}$ for orbit N. 15 with a decrease of the intensity $F$, within which the magnetic elements are widely variable with appreciable variances.

c) an outher region, starting at $22,7 R_{e}$, beyond which the magnetic elements become very stable and, above all, the variances are close to 
zero and below the sensitivity of the magnetometer $(0,25$ gammas $)$. A unique feature occurs at about $24,5 R_{e}$, where for a short time interval (10 to 30 minutes) an increased variance is apparent.

The boundary between the magnetosphere and the transition region is what we called the magnetopause; the boundary between the transition region and the outer and more quiet region identifies the collisionless shock wave of the solar plasma impinging on the outer magnetic field of the Earth. The small increase a few radii beyond the shock wave front is probably a "precursor" of the shock wave, in the sense that it may be an effect communicated "upstream" to the incoming solar plasma and magnetic field.

The deviations of the magnetic field intensity near and within the magnetopause are consistent with the characteristics of a compressed geomagnetic field. The field intensity is not very stable possibly because appreciable energy is transmitted into the magnetosphere as magnetohydrodynamic waves. Near the magnetopause the field is approximately tangential to its surface. At the subsolar point the average geocentric distance of the magnetopause is about $10 R_{e}$; the magnetic field intensity decreases very rapidly from 30-60 to 5-20 gammas in a few hundreds or one thousand $\mathrm{km}$. The fields on either side of the magnetopause are oppositely directed at times.

The collisionless shock wave boundary is observed experimentally at $13,4 R_{e}$ at the subsolar point; its thickness is of the same order of the magnetopause. The field within this thickness is randomized and large fluctuations occur.

We can remark that the observation of collisionless shock waves is a unique feature of interplanetary magnetic measurements due to the impossibility of reproducing them in the proper scale by laboratory experiments.

Also the "precursor" mentioned above has a spatial width of some thousands kms.

The intensity of the interplanetary magnetic field is very stable, even though appreciable variability in its direction occurs. In general $\left({ }^{24}\right)$ the field lies (Fig. 9) a little below the ecliptic plane $\left(\vartheta=20^{\circ}\right.$ to $30^{\circ}$ ) at an angle $\bar{\varphi}=130^{\circ}$ to $150^{\circ}$ or, in the opposite sense, $\Phi=310^{\circ}$ to $330^{\circ}$ (the corresponding values of the streaming angle $\psi$ are $30^{\circ}$ to $50^{\circ}$ and $130^{\circ}$ to $150^{\circ}$, respectively).

The gross structure of the field is in rather good agreement with Parker's idea of a spiral field. Within small thickness boundaries between regions of oppositely directed fields, null intensities are observed; 
they are interpretable as "neutral" surface of zero field strength separating field filaments in opposite directions.

This point is illustrated by Fig. 10 which shows the sectorial configuration of the field $\left({ }^{25}\right)$. The signs indicate a field away from the Sun $(+)$ and toward the Sun (-). Four different sectors are present, corresponding to approximately $1 / 7$ and $2 / 7$ of $360^{\circ}$. This structure
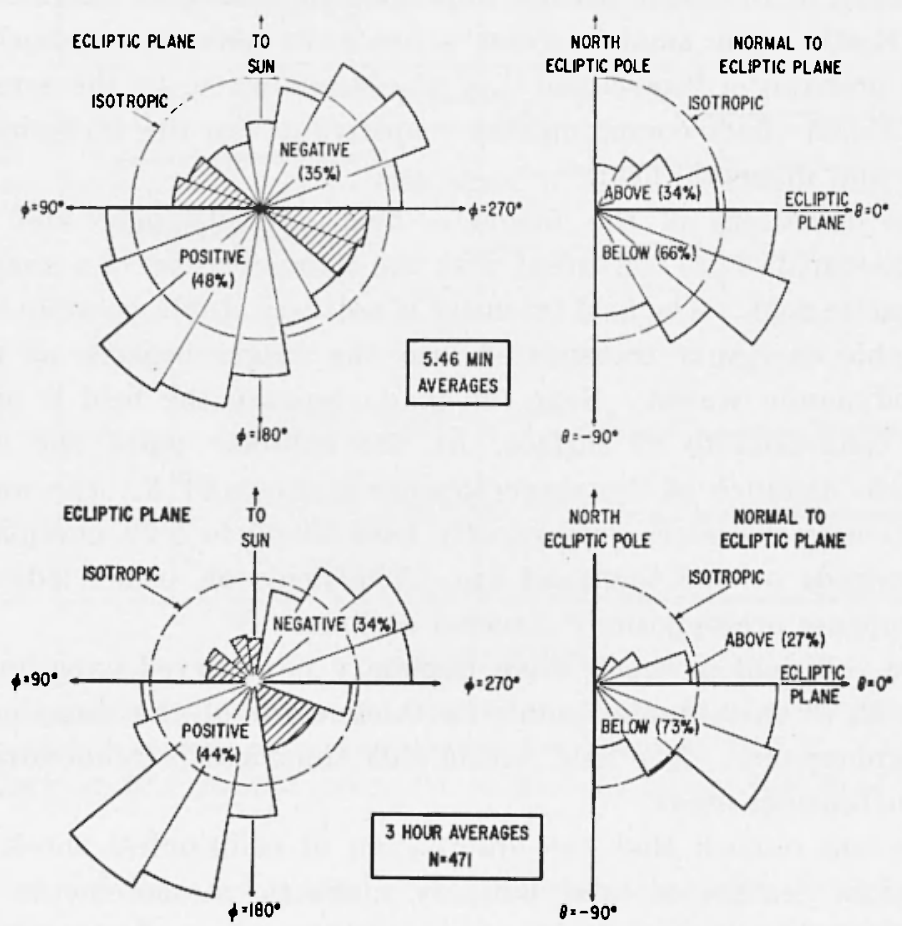

Fig. 9 - Distribution of interplanetary magnetic field directions (after Ness).

appears as quasi-steady during the three solar rotations the satellite could use to measure the interplanetary field. The tendency to a 27 day recurrence of the field characteristics is elearly shown by the autocorrelation of the observed direction of the interplanetary field (Fig. 11).

Another point of interest is the correlation between the surface magnetic field on the Sun (the photospheric field) and the measured interplanetary field ${ }^{(26)}$. Fig. 12 shows the photospheric field on the center of the solar disk for each day. The correlation of such field with the interplanetary field shown in Fig. 13 has a peak when the delay 


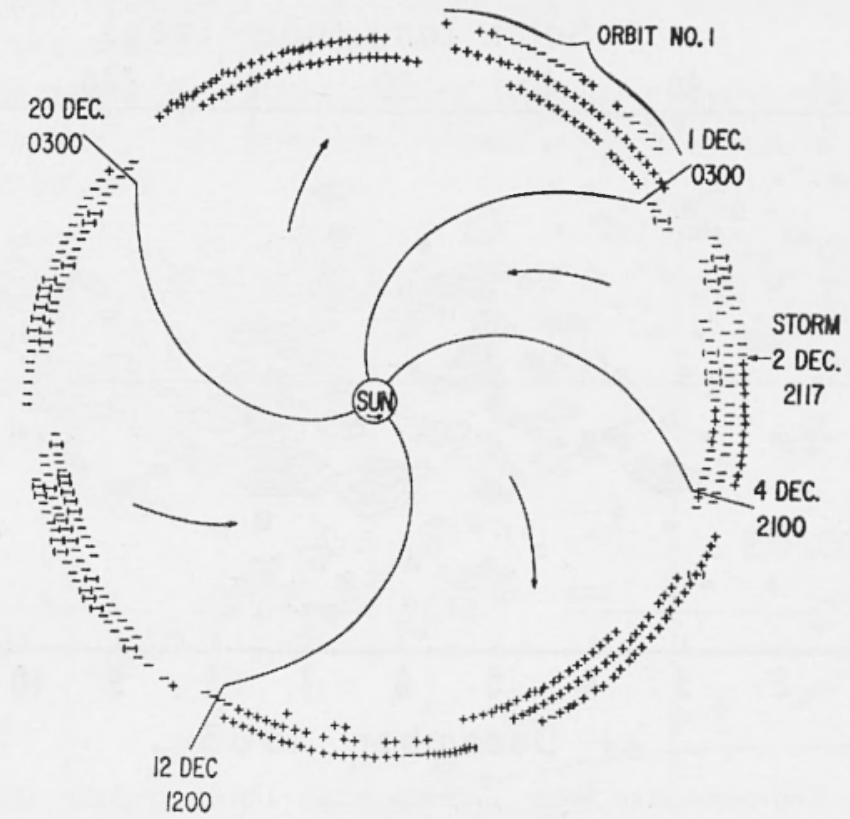

Fig. 10 - Sectorial distribution of the interplanetary magnetic field. Direction of magnetic field in each sector is indicated (after IVilcox and Ness).

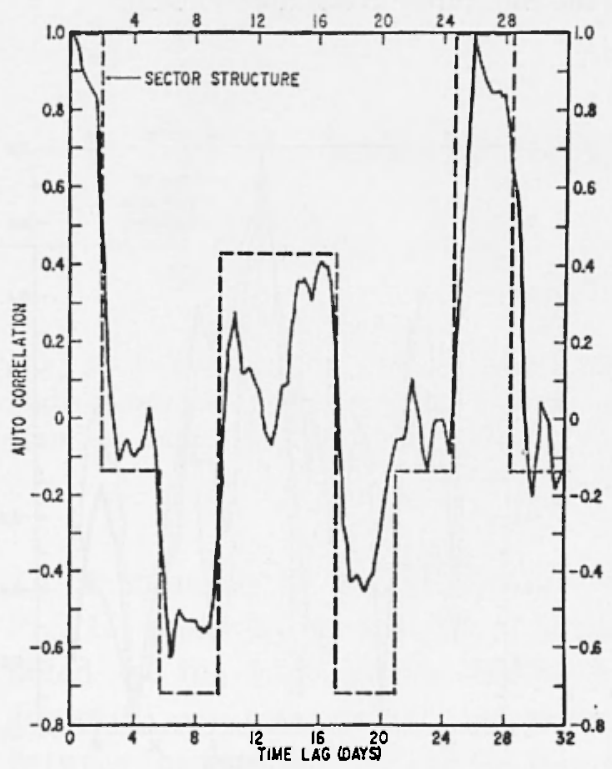

Fig. 11 - Time behavior of the autocorrelation coefficient for the interplanetary magnetic field (after Wilcox and Ness). 


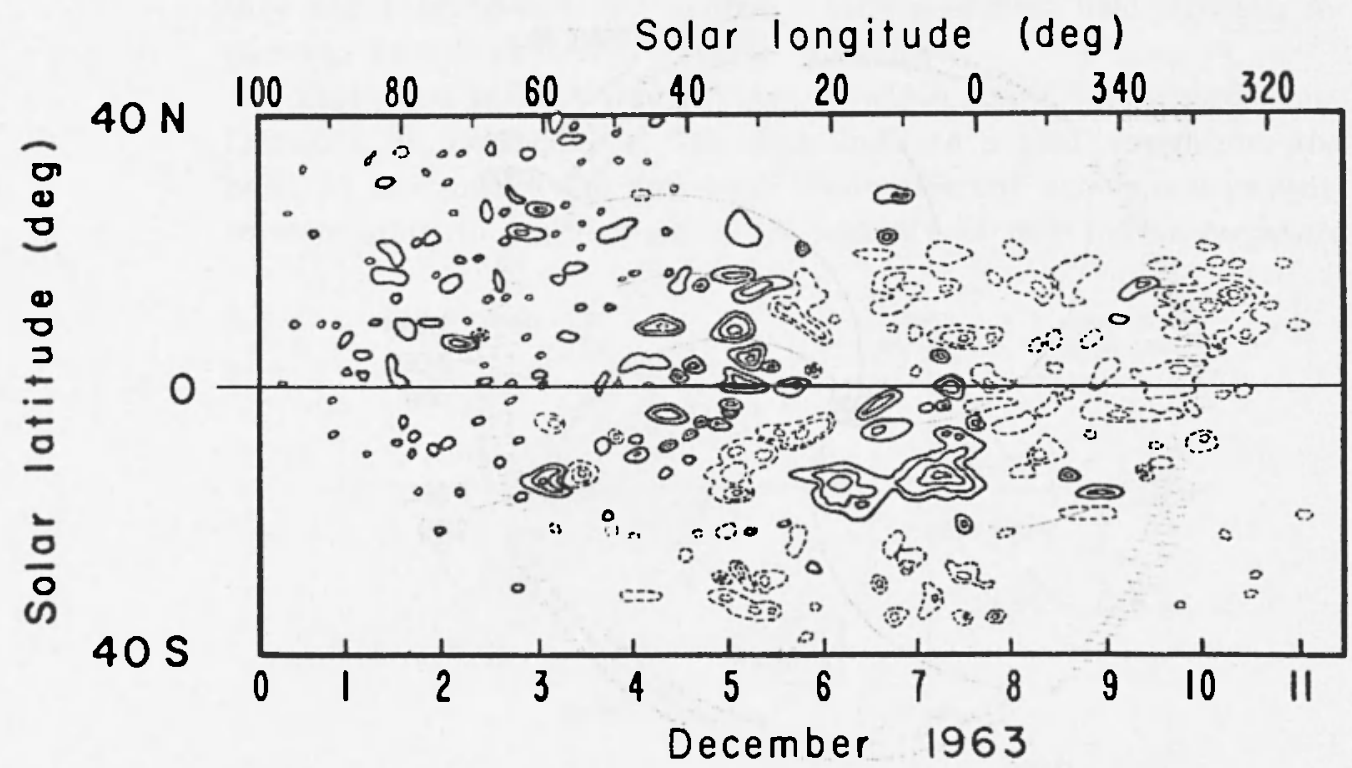

Fig. 12 - Representative solar magnetograph obtained from the Mount Wilson Observatory showing the magnitude and direction of the line-of. sight component of the photospheric magnetic field at CMP on date shown. The contour interval for these data are taken to be $2,4,8,12$ and 25 gauss. A solid line indicates fields directed out of the Sun while dashed lines indicate fields directed into the Sun (after Ness and Wilcox).

Fig. 13 - Cross correlation of the IMP-1 interplanetary magnetic field data and the photospheric magnetic field for three latitudes (after Ness).

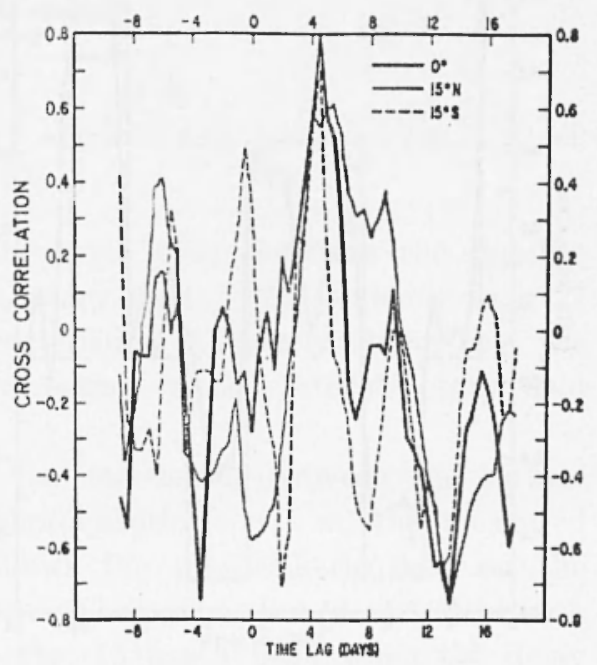


is 4,5 days which gives a figure of $385 \mathrm{~km} / \mathrm{sec}$ for the transport velocity of the photospheric field.

The geocentric distance of both the magnetopause and the shock wave boundary vary away the subsolar point, although by a different amount for each.

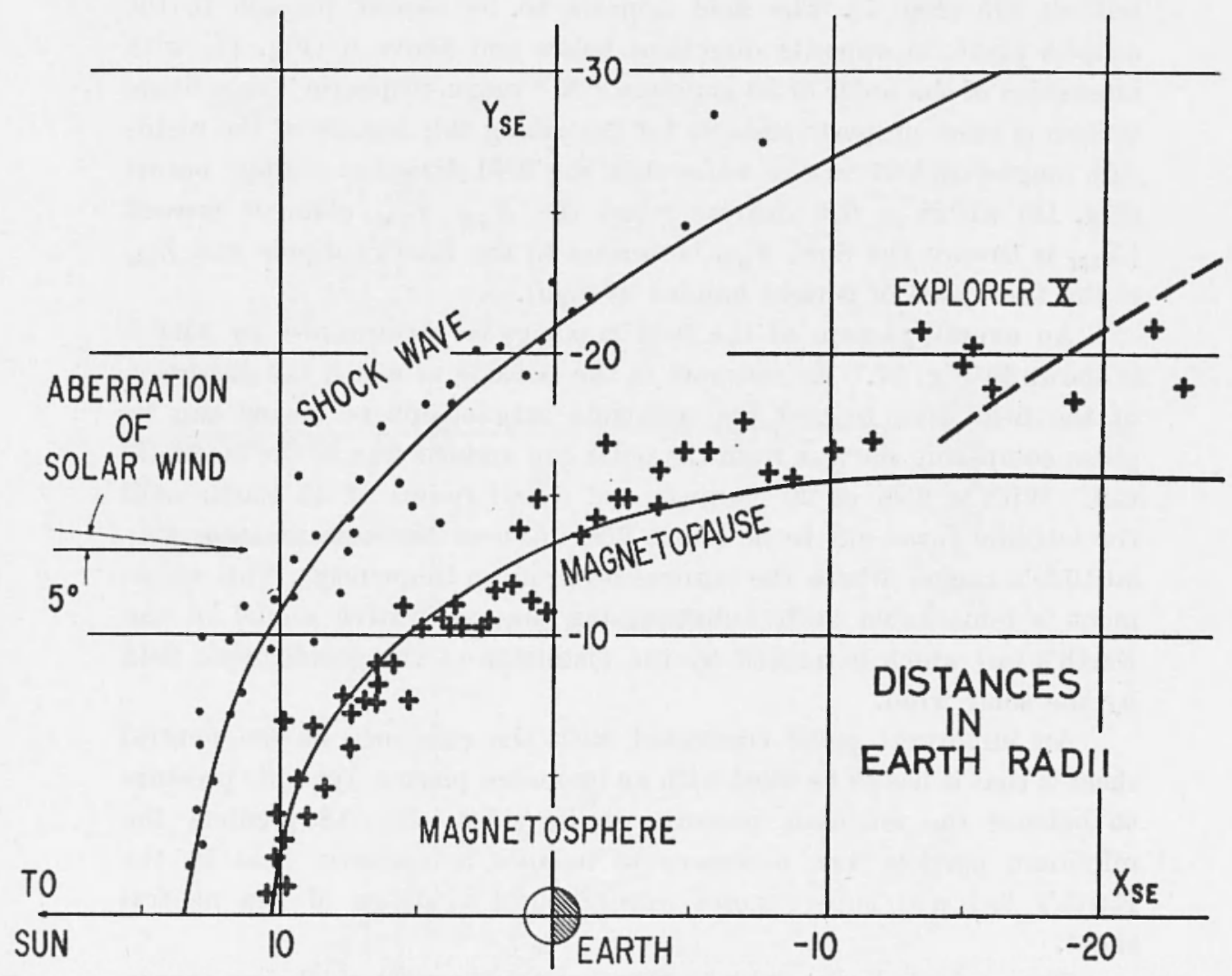

Fig. 14 - Comparison of the IMP-1 rectified boundary crossing with the high speed gas dynamic shock model of Spreiter and Jones (1962). The standoff ratio has been adjusted to match the observed measurements (after Ness et al.).

A summary of the location of both the boundaries is shown in Fig. 14, projected on the $X_{S E} Y_{S E}$ plane. Theoretical locations computed on the basis of the supersonic fluid model by SPREITER and JoNes (2?) are also given. An analogy can be actually established between the gasdynamics and the flow of plasma around the geomagnetic 
field $\left(^{28}\right)$. The agreement between theoretical inferences and experimental observations is very good.

A further important achievement of the IMP-1 mission was the exploration of the so-called magnetic tail of the Earth, i.e. the antisolar magnetosphere. This occurred in the last three months of satellite's life (Fig. 7). The field appears to be almost parallel to the ecliptic plane, in opposite directions below and above it (Fig. 15) with intensities of the order of 20 gammas. A "magnetospheric" coordinate system is more properly suitable for describing this feature of the nightside magnetosphere in the sense that the field direction change occurs (Fig. 16) within a few degrees when the $X_{S M} Y_{S M}$ plane is crossed ( $X_{S M}$ is toward the Sun; $Y_{S M}$ is normal to the Earth's dipole and $Z_{S M}$ is the third axis of a right-handed system).

An overall picture of the field topology as determined by IMP-1 is shown in Fig. 17. An estimate of the latitude at which the distortion of the field lines toward the nightside magnetosphere begins can be given comparing the flux from the polar cap and the flux in the magnetic tail. With a field of 20 gammas and a tail radius of 22 Earth radii the latitude turns out to be about $75^{\circ}$, i.e. near the auroral zones (the latitude's ranges where the aurorae occur more frequently). This agreement is remarkable as it substantiates the qualitative model fof the Earth's tail which is formed by the distorsion of the geomagnetic field by the solar wind.

An important point connected with the existence of the neutral sheet is that it has to be filled with an increased plasma dynamic pressure to balance the magnetic pressure on its sides. Fig. 18 predicts the minimum particle flux necessary to balance a magnetic field in the Earth's tail and superimposes experimental evidence of the neutral sheet.

The neutral sheet might be the channel through which low energy solar plasma particles enter the Earth's magnetic field.

Finally, a very interesting fact was observed during orbit N. 5 when the satellite was located between 28 and $31 R_{\varepsilon}$, far beyond the shock wave location. Fluctuations of the magnetic field elements and corresponding variances, very similar to those observed when sampling the transition region occurring between the magnetopause and the shock wave front, were observed. A possible fascinating interpretation is that the satellite was penetrating a magnetospheric cavity produced by the Moon (Fig. 19): the apparent length and diameter, expressed in lunar radii $R_{m}$ are at least $150 R_{m}$ and $30 R_{m}$, respectively. 


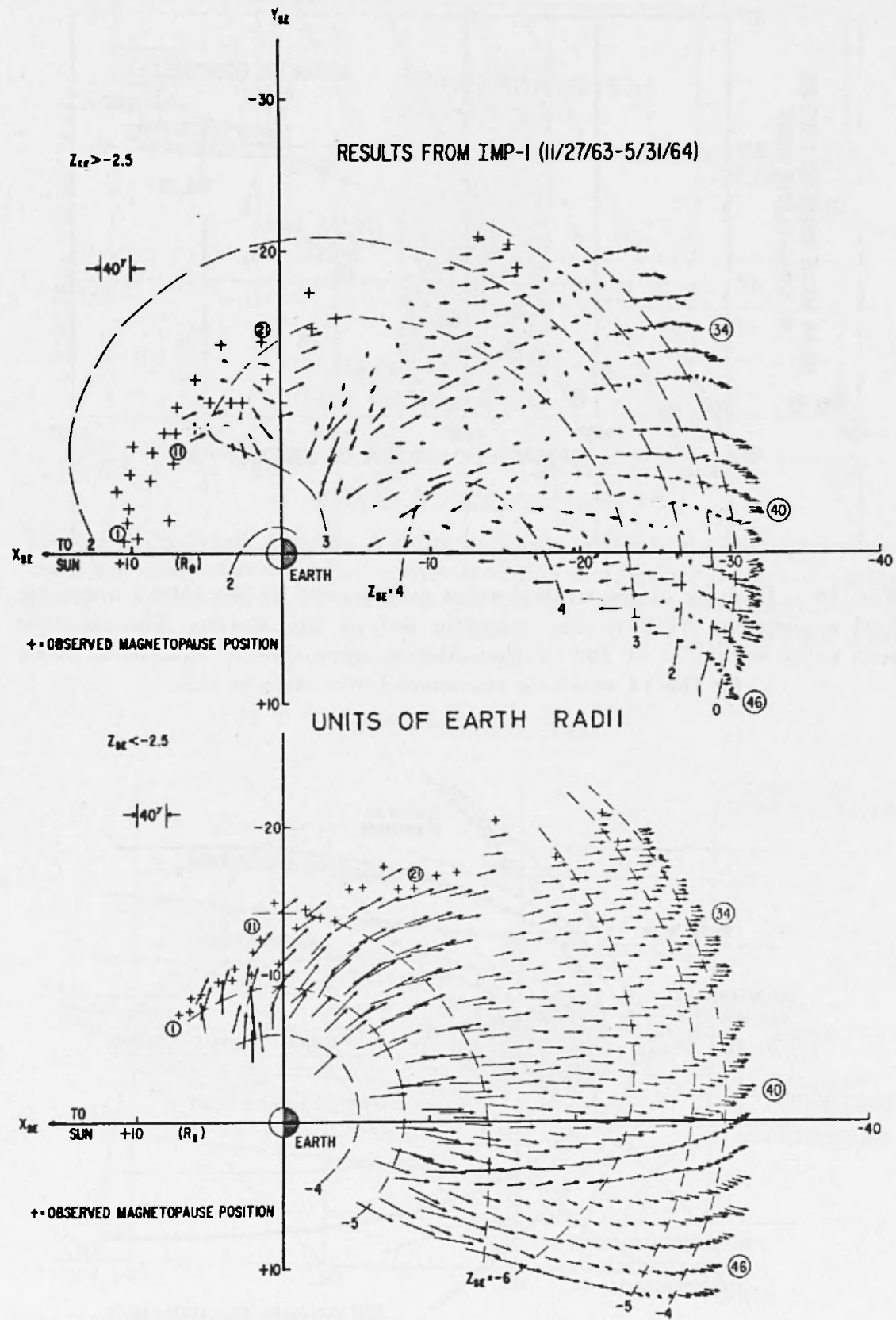

Fig. 15 - Magnetic field orientation in the nightside magnetosphere above and below the solar ecliptic (after Ness et al.). 


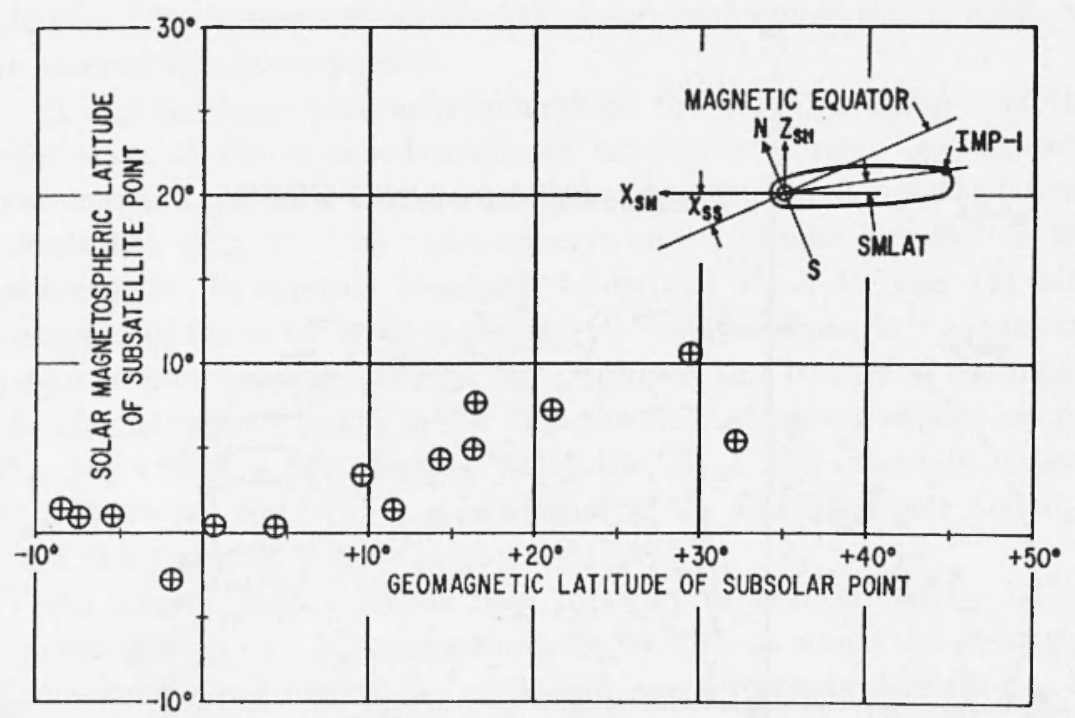

Fig. 16 - Position of the neutral sheet as observed by the lMP-1 magnetic field experiment while in the magnetic tail of the Earth. The sheet is seen to be within $5^{\circ}$ or $10^{\circ}$ of the solar magnetospheric equatorial plane for the 14 available traversals (after Ness et al.).

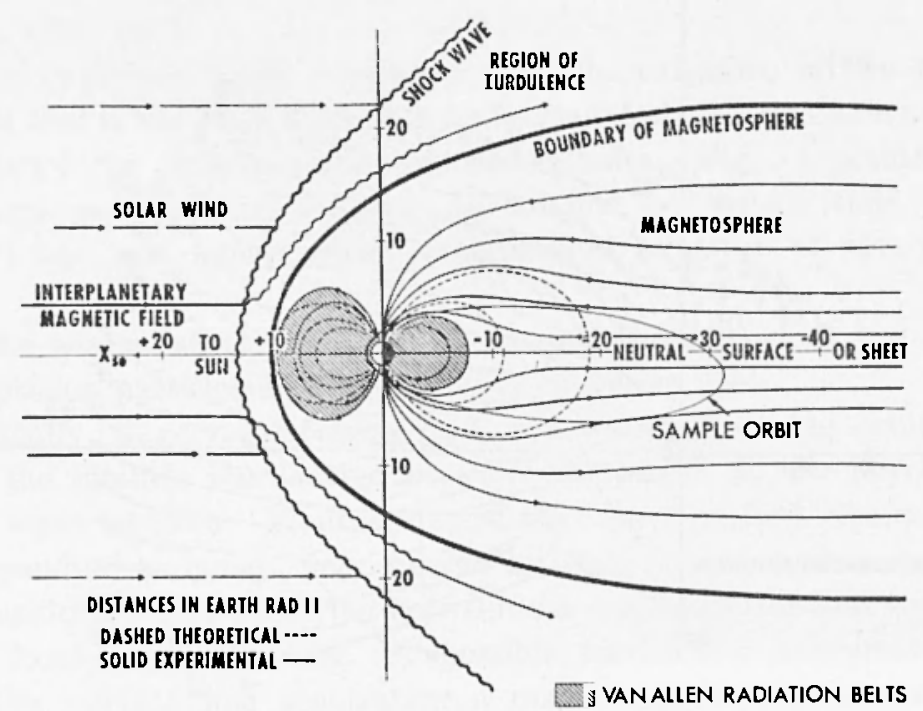

Fig. 17 - Projection of interplanetary magnetic field topology aecording to IMP-1 on noon-midnight meridian (after Ness). 


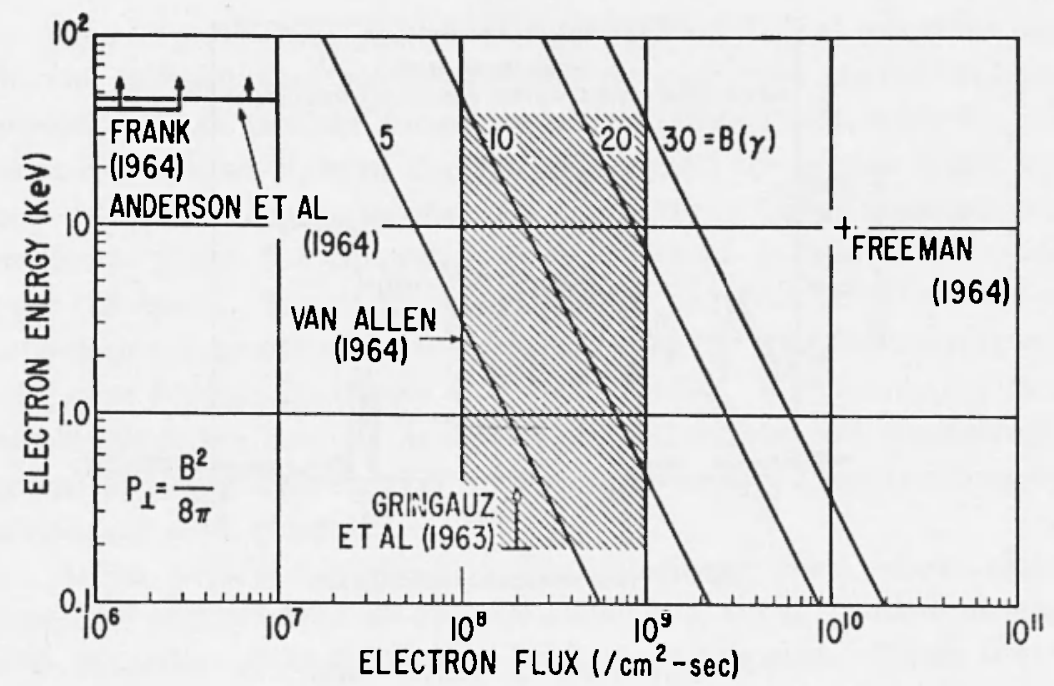

Fig. 18 - Assumed pressure balance between vacuum magnetic fields and nonmagnetized electron plasma corresponding to idealized model of neutral sheet. Superimposed are particle and plasma observations pertinent to the interpretation of the neutral sheet in the Earth's nagnetic tail (after Ness et al.).

VIEW ON ECLIPTIC PLANE

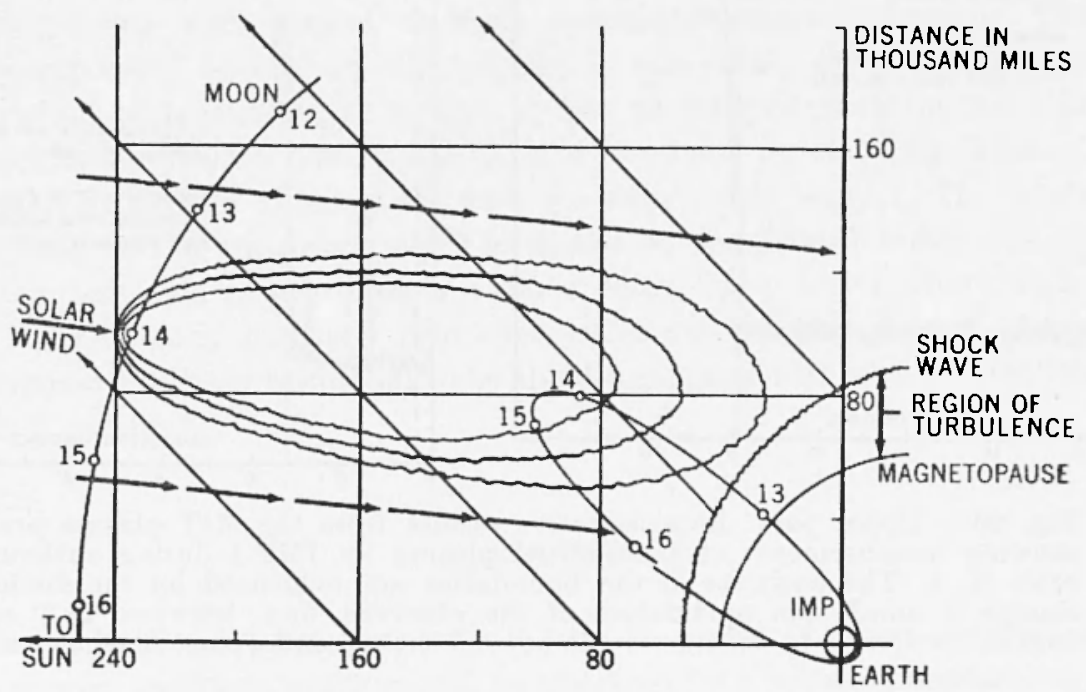

Fig. 19 - Interpretation of IMP-1 magnetic field data indicating characteristics of interplanetary and cislunar space and wake of Moon at time of lunar magnetosphere detection (after Ness et al.). 


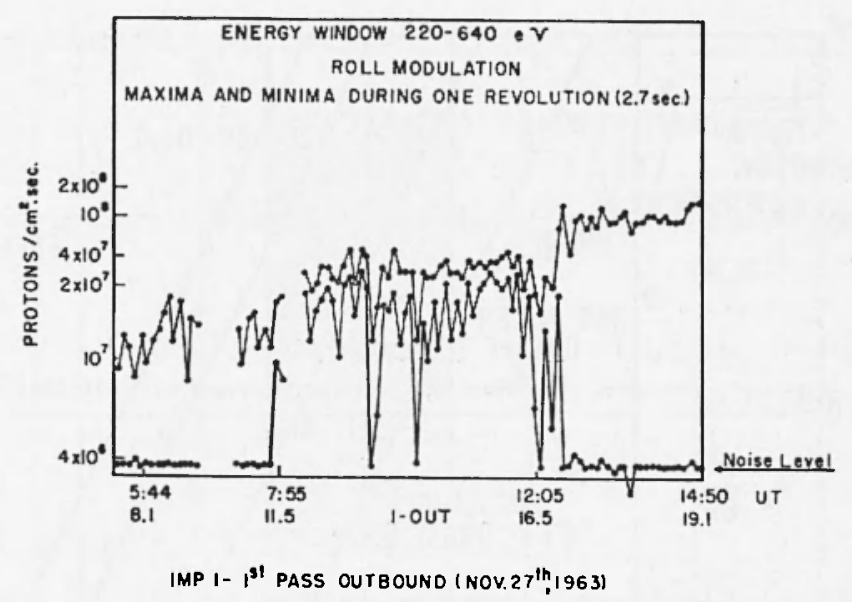

DISTANCES IN EARTH RADII
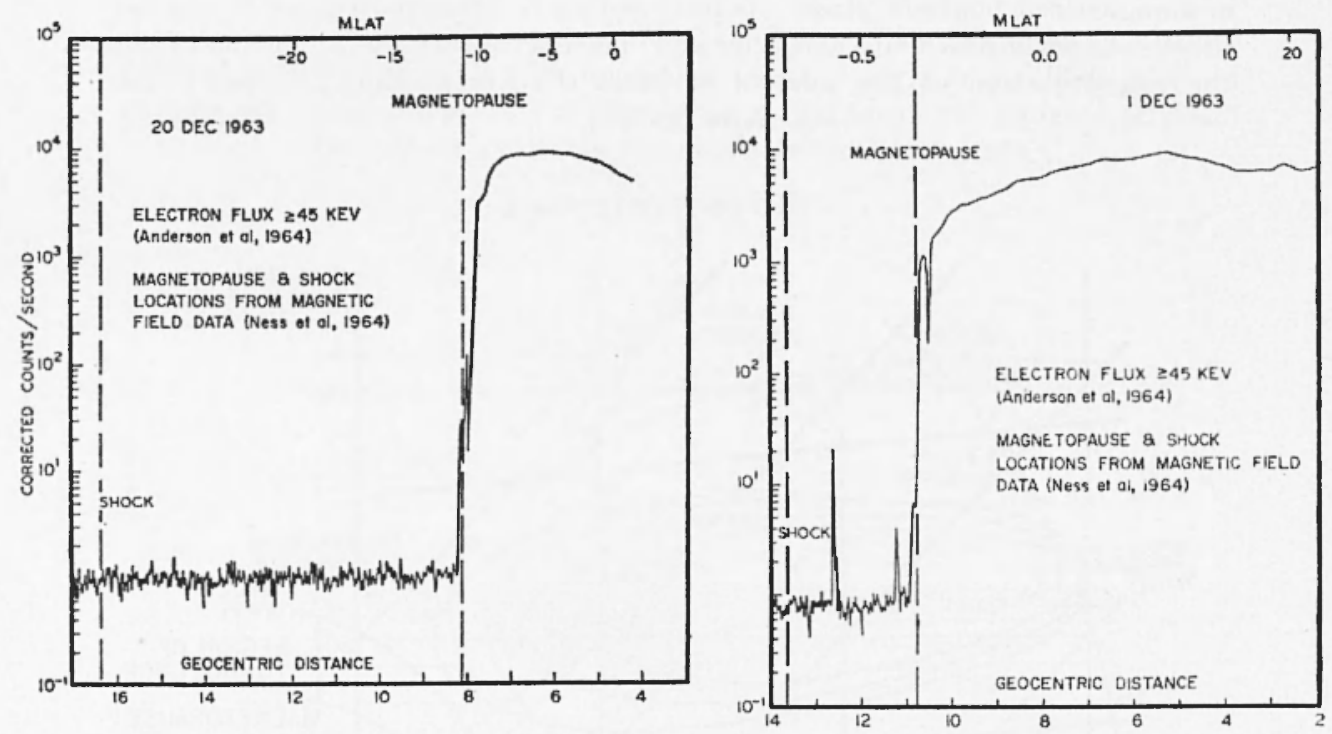

Fig. 20 - Upper part: Representative results from the MIT plasma probe showing measurements of thermalized plasma by IMP-1 during outbound orbit N. 1. The positions of the boundaries are evidenced by the distinct change to small spin modulation of the observed flux between 11,3 and 16,8 $R_{e}$ compared to measurements beyond these points (after Bridge et al.); Lower part:

- Left: Flux of electrons with energy greater jthan $45 \mathrm{keV}$ on inbound orbit N. 6 by the IMP-1 satellite.

- Right: Flux of electrons with energy greater that $45 \mathrm{keV}$ on IMP-1 inbound orbit $\mathrm{N}$. I (after Anderson et al.). 
The experimental picture we gave above is not complete if we do not mention that the information derived from plasma and, more generally, from particle measurements is substantially coherent. Just as an example we show in Fig. 20 (upper part) the proton fluxes measured $\left({ }^{29}\right)$ on IMP-1 in the energy window 220 to $640 \mathrm{eV}$ : maximum and minimum fluxes for one revolution are shown as a function of time (and distance). The region between 11,3 and $16,8 R_{e}$ where the spin modulation is practically zero corresponds to the transition region where the solar plasma undergoes its thermalization. At distances below and above them the flux is highly directional, within the magnetosphere (trapped particles) and outside in the interplanetary space (unidirectional streaming solar plasma).

Other evidence is given in Fig. 20 (lower part) where electron fluxes for energy above $45 \mathrm{keV}$ are shown $\left({ }^{30}\right)$; a 3 to 4 orders of magnitude reduction of the flux occurs at the magnetopause. These electrons are trapped inside the magnetosphere just until its boundary.

\subsubsection{Mariner 2 .}

Measurements at a distance of the order of 1 A.U. are those by Mariner 2. Unfortunately, they are contamined by a large ( $100 \mathrm{gam}$ mas) magnetic field of the spacecraft. The variations of the three components with respect to their averages $\left({ }^{31}\right)$ were computed. The $x$-component, normal to the ecliptic, is zero when averaged over an interval of several ditys, so that it can be inferred that the variable part of the field is mainly due to a vector lying on the ecliptic plane, making an angle of about $45^{\circ}$ with the solir radius vector. This angle is near to the streaming angle predicted by the Parker model. A 27 days variation is also clearly evident from the $y$ and $z$ components.

The steady magnetic field over which the variable field is superimposed has been estimated to be about $\mathbf{5}$ gammas with a rms deviation of 2 gammas.

\section{CONCLUSIONS}

Before concluding our talk it can be useful to point out just a few open questions which should be answered for a better understanding:

(i) an extensive mapping of the magnetic field into and outside the magnetosphere. Use of two or more satellites properly spaced 
would give direct informations about geometric - and time - scales. In particular the field above polar caps should be investigated in order to establish if the magnetosphere is "closed" or "open" (in the sense that polar lines of force extend to infinity, as Dungey thinks). More particularly the neutral points, if any, should be identified.

(ii) Correlation between surface field on the Earth and interplanetary field has to be studied to localize possible electric currents in the magnetosphere. Even the actual origin of auroral phenomena, radiation belts and some typical VIF phenomena should be investigated to yield informations about particle-wave interactions and acceleration mechanism.

(iii) Study of time (and space) magnetic field oscillations should be made to get informations on its non-steady components.

\section{BIBLIOGRAPHY}

(1) Chapman S., Bartels J., The Sphericar Harmonic Analysis of the Main Field. "Geomagnetism ", 2, 639-688, Oxford Univ. Press (1940).

( $\left.{ }^{2}\right)$ Finch H. F., Leaton B. R., The Earth's Main Magnetic Field, epoch 1955. "Monthly Notices Roy. Astr. Soc., Geoplys. Suppl.", 7, 314. 317 (1957).

(3) Jensen D. C., Whitaker W. A., A Spherical Harmonic Analysis of the Geomagnetic Field. April 1960 A.G.U. Meeting. "J. Geoplyys. Res. ", 65, 2500 (1960) and AFSWC Report, Kirtland AFB, New Mexico.

(1) Jensen D. C., CaIn J. C., An Interim Geomagnetic Field. April 1960 A.G.U. Meeting. "J. Geophys. Res.", 67, 3568 (1962) and Contract NAS 5-1278 (1962).

(5) Heuring F. T., The Analylic Description of the Geomagnetic Field at Satellite Altitude. "J. Geophys. Res.", 69, 4959-4968 (1964).

(") Smith E. J., Davis L., Coleman P. J., Sonetт C. P., Mariner 2: Preliminary Reports on Measurements of Tenus. "Science", 139, 909. 910 (1963).

(7) Drake F. D., Radio Emission from the Planets. "Physics Today" 14, 30-34, N. 4, April (1961).

(8) Dolginov S. SH., Eroshenko E. G., Zhuzgov L. N., Tyurmina L. O., Measuring the Magnetic Fields of the Earth and Moon by means of Sputnik 3 and Space Rockets 1 and 2. "Space Research"1, 863-868 (1960). 
(9) Baвсоск H. W., Вавсоск H. D., The Sun's Magnetic Field, 1952-1954. "Ap. J. ", 121, 349-366 (1955).

(10) HaLe G. E., General Magnetic Field of Sun. "Ap. J." 38, 27-98 (1913).,

(11) Parker E. N., Dynamical Instability in an Anisotropic Gas of Low Density. "Phys. Rev.", 109, 1874-1876 (1958).

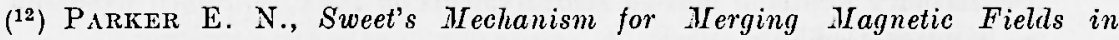
Conducting Fluids. "J. Geophys. Res.", 62, 509-520 (1957).

(13) Cocconi G., Greisen K., Morrison P., Gold T., IIayakawa S., The Cosmic Ray Flare Effect. "N. Cimento Suppl.", 8, 161-168 (1958).

(14) Piddington J. H., Interplanetary Magnelic Field and its Control of Cosmic Ray Variations. "Phys. Rev.", 112, 589-596 (1958).

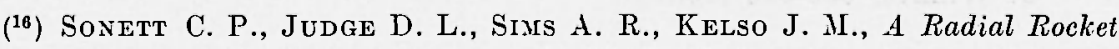
Survey of the Distant Geomagnetic Field. " J. Geophys. Res. ", 65, 55-68 (1960).

(16) Coleman P. J. Jr., Sonetr C. P., Judge D. L., Sarth E. J., Some Preliminary Results of the Pioneer $\mathrm{V}$ Magnetometer Experiment. " J. Geophys. Res.", 65, 1856-1857 (1960).

(17) Coleman P. J. Jr., Davis L., Soxetr C. P., Steady Component of the Interplanelary Magnetic Field: Pioneer V. "Phys. Rev. Letters", 5, $43.46(1960)$.

(18) Greenstadt E. W., Magnetic Storm in Interplanetary Space as Observed by Pioneer $V$. "Nature ", 191, 329-331 (1961).

(19) Greenstadt E. W., Moreton G. E., A comparison of Solar Flare Incidence with Magnetic Transients Observed in the Near-by Interplanetary Region by Pioneer F. "J. Geophys. Res.", 67, 3299-3316 (1962).

(20) IIeppner J. P., Ness N. F., Scearce C. S., Skildman T. L., Explorer 10 Magnetic Field Measurements. "J. Geophys. Res. ", 68, 1-46 (1963).

(21) Cainill L. J., Amazeen P. G., The Boundary of the Geomagnetic Field. "J. Geophys. Res. ", 68, 1835-1843 (1963).

${ }^{(22)}$ Camill L. J., The Geomagnetic Field, in Space Physics (edited by J. VILEY), 301-349 (1964).

$\left({ }^{23}\right)$ Ness N. F., Scenrce C. S., Seen J. B., Initial Results of the IMP-1 ILagnetic Field Experiment. "J. Geophys. Res, ", 69, 3531-3570 (1964).

${ }^{(24)}$ Ness N. F., WILcox J. M., On the Solar Origin of the Interplanetary Magnetic Field. "Phys. Rev. Letters", 13, $461-464$ (1964).

${ }^{(25)}$ Ness N. F., WILcox J. M., Sector Structure of the quiet interplanelary magnetic field. N.A.S.A. Report X, 612, 65-157.

$\left({ }^{26}\right)$ Ness N. F., WILcox J. M., Extension of the pholospheric magnetic field into interplanelary space. N.A.S.A. Report X, 612, 65-79.

(27) Spreiter J. R., Briggs B. R., Theoretical Determination of the Form of the Boundary of the Solar Corpuscular Stream Produced by Interaction 
with the Magnetic Dipole Field of the Earth. "J. Geophys. Res.", 67, 37-51 (1962).

(28) Levy R. H., Perscinek H. E., Siscoe G. L., Aerodynamic Aspects of the Magnetosphere Flow. AVCO Corp. Res. Rep. 170 (1963).

(29) Bridge H. A., Egidi A., Lazarus A., Lyon E., and Jacobson L., Preliminary results of plasma measurements on IMP-A. Space Research 5, 969.978 (1965).

(30) Anderson K. A., Harris H. K. and Paoli R. J., Energetic electron Auxes in and beyond the Earth's outer Magnetosphere. "J. Geophys. Res. ", 70, 1039-1050 (1965).

(31) Surmi E. J., Interplanetary Magnetic Fields, in Space Plysics (edited by J. WILEY), 350-396 (1964).

A description of experimental tecniques for magnetic field measurements and related bibliography are given in the ESRO Scientific Report n. 4 (Magnetic field measurements in interplanetary space) by S. Cantarano and F. Mariani (1965). 